\title{
PRÁCTICA PEDAGÓGICA Y EXPERIENCIA PROFESIONAL: FORTALEZAS Y OPORTUNIDADES DE MEJORA
}

\section{PEDAGOGICAL PRACTICE AND PROFESSIONAL EXPERIENCE: STRENGTHS AND OPPORTUNITIES FOR IMPROVEMENT}

\section{Yuretsy Alexandra Reyes Cáceres ${ }^{1}$}

\section{Raúl Prada Núñez ${ }^{2}$}

Audin Aloiso Gamboa Suárez ${ }^{3}$

\section{RESUMEN}

En el presente artículo de investigación se muestran las fortalezas y oportunidades de mejora en las prácticas pedagógicas de maestros de matemáticas en 15 instituciones educativas de Norte de Santander. La metodología

Semillero de Investigación en Educación Matemática, Grupo de Investigación en Pedagogía y Práctica Pedagógica, Licenciatura en Matemáticas, Universidad Francisco de Paula Santander. E-mail: yuretsyalexandrarc@ ufps.edu.co. ORCID: 0000-0001-6862-8417.

$2 \quad$ Magister en Ingeniería de análisis de datos, mejora de procesos y toma de decisiones. Docente investigador Universidad Francisco de Paula Santander. E-mail: raulprada@ufps.edu.co. ORCID: 0000-0001-6145-1786.

3 Doctor en Ciencias de la Educación por la Universidad de Cartagena (Colombia). Docente investigador de la Universidad Francisco de Paula Santander. Correo electrónico: audingamboa@ufps.edu.co. Orcid: 0000-00019755-6408. adoptada se ajusta a las características del enfoque cuantitativo, a nivel descriptivo con un diseño de campo. Los integrantes de la muestra son un grupo de 80 docentes que laboran en instituciones públicas y privadas de Cúcuta y su área metropolitana. Se aplicó un cuestionario que ha sido validado y se compone de 34 ítems evaluados mediante una escala Likert de cinco niveles. Los resultados permitieron identificar que todos los aspectos que envuelven la práctica pedagógica se correlacionan significativamente entre ellos. Se concluye que los docentes en sus primeros años de experiencia no realizan ningún estudio posgradual, sino que se dedican solo a trabajar y satisfacer sus necesidades familiares y económicas. 


\section{PALABRAS CLAVE:}

práctica pedagógica, experiencia docente, fortalezas, oportunidades de mejora

\section{ABSTRACT}

This research article shows the strengths and opportunities for improvement in the pedagogical practices of mathematics teachers in 15 educational institutions in Norte de Santander. The methodology adopted conforms to the characteristics of the quantitative approach, at a descriptive level with a field design. The members of the sample are a group of 80 teachers who work in public and private institutions in Cúcuta and its metropolitan area. A questionnaire was applied that has been validated and is composed of 34 items in total corresponding to the items of teaching practice that are evaluated using a five-level Likert scale. The results allowed to identify that all the aspects that involve the pedagogical practice are significantly correlated between them. It is concluded that teachers in their first years of experience do not carry out any postgraduate study, but rather dedicate themselves only to working and satisfying their family and economic needs.

\section{KEYWORDS:}

pedagogical practice, teaching experience, strengths, opportunities for improvement

\section{INTRODUCCIÓN}

Al pasar los años continua la preocupación de que se otorgue una educación de calidad a los estudiantes, por ende, se han propiciado diversos estudios y cambios drásticos que han influido en el proceso de enseñanza (Pacheco y Ramos, 2015; Gamboa, 2016; Lago, Gamboa, Montes, 2014). Generando grandes retos a los docentes que orientan el área de matemáticas, en donde este debe actuar como orientador e investigador, analizando e interpretando todas aquellas situaciones que presencia en el aula de clase, a partir de su reflexión busca estrategias que propicien una solución a todos los problemas a los cuales se enfrenta a diario (Rodríguez, 2010). El docente juega un papel importante en el proceso de enseñanza y aprendizaje, pues la intervención del docente permite responder a las necesidades de la sociedad actual (Piñero, 2020; Penagos, Mariño y Hernández, 2017).

Para lograr una educación de calidad el docente debe ser un líder y mediador entre la sociedad y el conocimiento, siendo un ejemplo a seguir para sus estudiantes. ¿por qué se hace tanto énfasis en el papel docente? es evidente que el principal agente educativo es el docente y en la pedagogía ha jugado un papel fundamental (MinEducacion, 2005). Históricamente el docente es generador de cambio, de transformación para el proceso de enseñanza- aprendizaje. El papel del docente es fundamental en la educación tal como lo afirman Basurto, Hernández y Rodríguez (2013).

Los docentes, como profesionales de la educación, deben potenciar las capacidades intelectuales de los estudiantes, propiciar aprendizajes significativos, favorecer el desarrollo del pensamiento crítico y científico e intervenir para adquirir nuevas formas de convivencia democrática en el aula multicultural y diversa, asumiendo así la responsabilidad de desarrollar en los estudiantes las competencias que son necesarias para continuar aprendiendo a lo largo de la vida (p.1).

Es por ello que el docente cobra gran importancia a los efectos que contraigan la reflexión, innovación y sentido crítico para satisfacer las necesidades de este mundo cambiante que demanda su práctica docente. La práctica pedagógica es entendida como aquellos aspectos que el docente realiza para que el estudiante adquiera una formación integral (Duque, Vallejo \& Rodríguez, 2016; García-Quintero \& Villamizar-Suárez, 2017). 
Para lograr esto el docente debe mantener comunicación con toda la comunidad educativa, transmitir, evaluar y socializar cada una de las experiencias vivenciadas obteniendo de esto la reflexión de su práctica docente. la constante reflexión sobre su práctica pedagógica es fundamental en el proceso educativo porque permite que los docentes realicen una revisión sobre sus aciertos y desaciertos que se encuentre en el proceso de enseñanza, conllevando a que el docente logre orientar el mejoramiento de la educación hacia un camino de calidad. En las prácticas pedagógicas se encuentran inmersos muchos aspectos tales como: la preparación de materiales, actividades y recursos requeridos para el aprendizaje del estudiante, la organización y fundamentación del aula, entre otras.

Al momento de hablar de las oportunidades de mejora presentes en el aprendizaje de las matemáticas intervienen muchos factores presentes en la práctica pedagógica que no son solo generados por parte de los estudiantes si no de los mismos docentes. Según Chevallard (como se citó en Morillo y Valencia, 2014) la práctica pedagógica es "la capacidad que tiene el docente en transformar el saber que posee (científico) al saber posible de ser enseñado, en el cual el docente realiza una despersonalización de su conocimiento de tal forma de que los educandos se apropien de él" (p.38). Tal como lo menciona el autor las prácticas docentes son de gran importancia porque determinan todas aquellas acciones que facilitan el proceso de aprendizaje del estudiante, en donde el docente transforma estos conocimientos científicos y emplea un lenguaje adecuado para que sus estudiantes creen estructuras mentales y se apropien de este conocimiento, es decir no solo se basa en brindar teoría, sino que a su vez los estudiantes construyan nuevos saberes, apliquen lo aprendido en situaciones de su cotidianidad y desarrollen con este competencias matemáticas.
Como lo dicho anteriormente la práctica pedagógica y todas sus dimensiones juegan un papel importante en la educación de calidad que se desea lograr y lo confirma Colorado (2012),

la práctica pedagógica es importante porque por medio de ella el maestro puede demostrar su destreza, actitudes y competencia para la dirección, control y evaluación del aprendizaje de los diferentes niveles del sistema educativo. Por esto para poder tener claridad la concepción de aprendizaje que subyace al programa de una buena instrumentación didáctica, es conveniente que el profesor cuente con elementos teóricos técnico que le permitan interpretar didácticamente un programa educacional a partir de una teoría y con una concepción de aprendizaje que lo lleve a propiciar en su educandos una formación acorde con el plan de estudio de la institución, el maestro enfrenta sus conocimientos pedagógicos al discurso o de las teorías de la ciencia y el instrumento que utiliza para ello es el método de enseñanza. (p.254)

Tal como lo expreso el autor la práctica pedagógica es importante tanto para el estudiante como para el docente, puesto que el docente demuestra las competencias logradas en su experiencia y así toma el control de los aprendizajes transmitidos a sus estudiantes, por ello en este proceso se ven involucrados y beneficiados ambos, el docente a través de la didáctica orienta su teoría siendo todas esas herramientas fundamentales para impartir el conocimiento de algún tema en específico, el proceso didáctico se da a través de las relaciones que tenga el estudiante en como enseñe el docente y como lo comprenda el estudiante para organizar esquemas mentales que le generen aprendizajes significativos y pueda relacionarlo con vivencias de su entorno. 
En este sentido el reflexionar sobre la práctica pedagógica de los docentes permite identificar aciertos y desaciertos del proceso de enseñanza en el aula de clase, generando gran impacto en la labor del docente y conllevando a la educación a un proceso de cambio, en esta reflexión el docente explora sus bases didácticas determinando si existe alguna creencia u acción indebida en su enseñanza (Martínez,2017) , es aquí donde el docente realiza una inspección introspectiva explorando todas aquellas características del contexto que son fundamentales para obtener un mejor conocimiento del entorno que rodea a los estudiantes y favorecer los lazos de comunicación entre docente-estudiante y la comunidad en sí misma (Pérez y Vallejo,2018). Las dimensiones de la práctica pedagógica son fundamentales en el quehacer docente que se deben tener en cuenta para emplear todos esos aspectos en la educación de calidad que se anhela.

Como se mencionaba anteriormente el MinEducación (2013), propone un marco profesoral en el cual el docente explora cuales son las características de un maestro excelente, identificando como se planifica una clase, como se aborda la enseñanza, como se generan ambientes propicios en el aula de clase y como es empleada la evaluación. Por ende, esta guía sirve para que los docentes realicen un ejercicio de autorreflexión y compara las prácticas de calidad que son consideras con las que emplea en su aula de clase. el marco profesoral propone 4 dimensiones

\section{PLANEACIÓN Y PREPARACIÓN DE CLASE}

La planeación y preparación de clase es uno de los pilares fundamentales para que el docente cumpla sus funciones como transmisor del proceso educativo. En las cuatro dimensiones fundamentales la planeación es una actividad de primer orden, siendo imprescindibles en la orientación y control para dirigir el proceso de enseñanza-aprendizaje, la planeación de clase es aquel hilo conductor de las herramientas didácticas, estrategias, estructura y acciones del contenido que se desarrolla tanto fuera como dentro del aula (Reyes, 2017).

Como lo dicho anteriormente, la planeación de clase es fundamental para que la labor del docente sea significativa, tal como lo afirma Zilberstein (2016), "La función de la planificación garantiza que el profesor pueda dirigir de manera científica el proceso de enseñanza aprendizaje. La planeación es una actividad creadora; mientras más se planee el proceso educativo, más seguridad se tendrá en su desarrollo y en el logro de los objetivos propuestos" (p.199). por ello, si se desea una educación de calidad el docente debe realizar una planeación de todo aquello que abordara en su aula de clase y es necesario que indague sobre las necesidades de los estudiantes y el entorno que lo rodea para facilitar el proceso educativo.

\section{AMBIENTES DE APRENDIZAJE}

Los ambientes generados en el aula de clase son fundamentales en el proceso educativo, puesto que es una habilidad esencial que el docente mantenga una buena relación en el aula de clase, generando un ambiente de empatía y respeto entre los estudiantes. Debido a la comunicación que exista los estudiantes se sentirán cómodos, seguros y valorados entre sus compañeros y docente. el docente sebe mostrar lo mejor de sí mismo, contribuyendo a través de sus prácticas a las necesidades de los estudiantes logrando así una educación de calidad.

Así mismo, al generar un ambiente propicio tanto docente como estudiantes en el interior del aula de clase establecen aquellas normas y reglas que conlleva al buen comportamiento en el aula. En relación con esto Ospina (como se citó en Duarte, 2003) afirma que "El ambiente es 
concebido como construcción diaria, reflexión cotidiana, singularidad permanente que asegure la diversidad y con ella la riqueza de la vida." (p.5). En conclusión, el ambiente de aprendizaje no solo se basa es factores afectivos también está involucrado en este proceso de enseñanza la infraestructura, los factores culturales, físicos y políticos que conllevan a propiciar un ambiente agradable para los estudiantes.

\section{PRÁCTICA PEDAGÓGICA}

Son muchos los aspectos que se encuentran inmersos en la práctica pedagógica, como se ha mencionado intervienen la planeación y organización de clase, preparación de material, recurso y herramientas adecuadas para el aprendizaje de los estudiantes. A partir de todo este proceso el docente logra enfrentarse a explorar sus capacidades con las que abordara su labor como docente. En este mismo sentido Freire (2011) expone:

La práctica educativa, es algo muy serio. Tratamoscongente, conniños, adolescentes o adultos. Participamos en su formación. Los ayudamos o los perjudicamos en esta búsqueda. Estamos intrínsecamente conectados con ellos en su proceso de conocimiento. Podemos contribuir a su fracaso con nuestra incompetencia, mala preparación o irresponsabilidad. Pero también podemos contribuir con nuestra responsabilidad, preparación científica y gusto por la enseñanza. (...). (p.52)

De acuerdo a lo anterior, se puede percibir que la labor docente es una ardua tarea en la cual tenemos en nuestras manos la formación integral y significativa de los estudiantes, si no se reflexiona sobre la práctica pedagógica no se podrá identificar las falencias presentes en el aula de clase, generando en los estudiantes deserción escolar, apatía, rechazo y frustración porque no logra comprender lo que el docente transmite.

\section{RESPONSABILIDADES PROFESIONALES}

Esta dimensión es fundamental en todo el proceso educativo, puesto que se destaca la labor que el docente tiene en contribuir a que los estudiantes obtengan aprendizajes significativos, es por ello que al reflexionar en su práctica pedagógica es consiente que si fortalece sus oportunidades de mejora está contribuyendo a garantizar una educación de calidad. De aquí surge la necesidad de comprender y descubrir las dificultades que se presenten, superando estas con la investigación de otras herramientas y estrategias de aprendizaje, también el docente está abierto a relacionarse no solo con estudiantes, sino con los demás docentes pertenecientes a la comunidad educativa, consientes en que se está en constante aprendizaje, además el docente es su calidad de mejorar acude a la actualización de herramientas, realizando otros estudios que lo ayuden a ampliar su conocimiento y adquiriendo experiencia (MinEducación, 2013)

\section{METODOLOGIA}

En esta investigación se ajustan las características del enfoque cuantitativo a nivel descriptivo correlacional y se caracteriza por ser secuencial y probatorio por que utiliza la recolección de datos para probar hipótesis con base en la medición y el análisis estadístico.

El instrumento utilizado fue un cuestionario que en primera instancia fue validado por juicio de expertos, el cual estaba integrado por tres docentes que vienen trabajando con el tema desde hace más de tres años, con una formación de pregrado y postgrado en matemáticas y con experiencia docente en educación básica secundaria, media y superior. La redacción de los ítems sufrió algunos cambios tras el análisis 
de sensibilidad cultural. Una vez se recolectaron los datos, se realiza la prueba de validación de constructos y confiabilidad recurriendo al software SPSS v25.

La población está conformada por la totalidad de docentes que laboran en 15 instituciones educativas públicas y privadas de Cúcuta y su área metropolitana, de los cuales se pudo obtener una muestra no probabilística de 80 docentes, y decimos que es no probabilística porque nos interesaban aquellos docentes que estuvieran orientando matemáticas. Es importante mencionar que en todo el documento se mantiene en anonimato la identidad de cada uno de los informantes. Cada docente diligencio de forma anónima el cuestionario que contaba de dos secciones, en la primera sesión contemplaban los datos sociodemográficos tales como: genero, formación académica, nivel máximo de formación, edad, años de experiencia, tipo de institución donde labora y donde está ubicada. En la segunda sesión se abarcaron los ítems relacionados a las practicas pedagógicas evaluados mediante una escala Likert con cinco niveles de cumplimiento (Nunca, Pocas veces, Algunas veces, Casi siempre y Siempre). En la tabla 1 se muestra la estructura del instrumento utilizado.

Tabla 1. Componentes del instrumento empleado.

\begin{tabular}{lc}
\hline \multicolumn{1}{c}{ Ítems evaluados } & Número de ítems \\
\hline Datos sociodemográficos & 7 \\
Planeación y preparación de clase & 11 \\
Ambientes de aprendizaje & 15 \\
Práctica pedagógica & 9 \\
\hline
\end{tabular}

Fuente. Elaboración propia

Una vez obtenidos los datos se procesaron y se utilizó estadísticas descriptivas y Chi-cuadrado para comparar si hay relaciones significativas entre las variables socio-demográficas y los ítems asociados a las practicas pedagógicas y entre los mismos ítems de la práctica pedagógica (planeación y preparación de clase, ambientes de aprendizaje y práctica pedagógica).

\section{RESULTADOS Y DISCUSION}

La práctica pedagógica es concebida como aquellas situaciones vivenciadas en el aula de clase que compone el quehacer del docente y estudiante. Según Avalos (2002) la práctica pedagógica es "el eje que articula todas las actividades curriculares de la formación docente, de la teoría y de la práctica" (p.109), por tanto, implica todas aquellas acciones tales como: organización y planeación de clase, preparación de materiales y recursos de aprendizaje acordes a las situaciones que vivencia el estudiante tanto fuera y dentro de aula. También en concebida como pruebas que supera el docente a medida que va adquiriendo experiencia y de aquellas actualizaciones que realiza para potenciar su conocimiento y capacidades. En la tabla 2 se especifica la valoración que dieron los docentes encuestados a un compilado de las dimensiones de la práctica pedagógica. 
Tabla 2. Compilado prácticas pedagógicas

\begin{tabular}{|c|c|c|c|}
\hline \multirow[b]{2}{*}{ Ítems } & \multicolumn{3}{|c|}{ Opciones de respuesta } \\
\hline & Nunca & $\begin{array}{l}\text { Algunas } \\
\text { Veces }\end{array}$ & Siempre \\
\hline $\begin{array}{l}\text { P1. Presenta a sus estudiantes de forma clara los } \\
\text { objetivos de la clase y las instrucciones de cada una de las } \\
\text { actividades a realizar. }\end{array}$ & $0.0 \%$ & $5.5 \%$ & $94.6 \%$ \\
\hline $\begin{array}{l}\text { P2. Presenta los conceptos con precisión, claridad e } \\
\text { imaginación utilizando el lenguaje académico adecuado. }\end{array}$ & $0.0 \%$ & $5.5 \%$ & $94.6 \%$ \\
\hline $\begin{array}{l}\text { P3. Al momento de diseñar las actividades de aprendizaje, } \\
\text { considera los diversos ritmos de aprendizaje de sus } \\
\text { estudiantes. }\end{array}$ & $0.0 \%$ & $8.2 \%$ & $91.8 \%$ \\
\hline $\begin{array}{l}\text { P4. Al momento de diseñar las actividades de aprendizaje, } \\
\text { considera las necesidades particulares de sus estudiantes. }\end{array}$ & $0.0 \%$ & $13.7 \%$ & $86.3 \%$ \\
\hline $\begin{array}{l}\text { P5. Al momento de planificar las clases, identifica } \\
\text { exactamente lo que espera que sus estudiantes aprendan. }\end{array}$ & $0.0 \%$ & $2.7 \%$ & $97.3 \%$ \\
\hline $\begin{array}{l}\text { P6. Impulsa a sus estudiantes a participar haciendo uso } \\
\text { del lenguaje preciso para profundizar en la comprensión de } \\
\text { conceptos. }\end{array}$ & $1.4 \%$ & $13.7 \%$ & $84.9 \%$ \\
\hline $\begin{array}{l}\text { P7. Motiva a los estudiantes a expresar sus puntos de vista } \\
\text { por medio del uso del lenguaje adecuado. }\end{array}$ & $1.4 \%$ & $8.2 \%$ & $90.4 \%$ \\
\hline $\begin{array}{l}\text { P8. Ocasionalmente, no proporciona toda la información } \\
\text { disponible con el fin de animar a los estudiantes a pensar } \\
\text { por sí mismo. }\end{array}$ & $8.2 \%$ & $34.2 \%$ & $57.5 \%$ \\
\hline $\begin{array}{l}\text { P9. Su planeación curricular refleja la secuencia de } \\
\text { procesos de aprendizaje garantizando el avance de los } \\
\text { estudiantes. }\end{array}$ & $0.0 \%$ & $15.1 \%$ & $84.9 \%$ \\
\hline $\begin{array}{l}\text { P10. La evaluación escrita es un recurso que permite } \\
\text { identificar los estudiantes que han alcanzado los resultados } \\
\text { obtenidos. }\end{array}$ & $2.7 \%$ & $4.1 \%$ & $93.1 \%$ \\
\hline $\begin{array}{l}\text { P11. La evaluación escrita es un recurso que permite } \\
\text { mejorar el proceso de enseñanza identificando las } \\
\text { debilidades del proceso de enseñanza. }\end{array}$ & $1.4 \%$ & $8.2 \%$ & $90.4 \%$ \\
\hline Porcentaje promedio & $1.0 \%$ & $10.8 \%$ & $87.8 \%$ \\
\hline
\end{tabular}

Fuente: Elaboración propia 
A nivel global el $87.8 \%$ de los docentes encuestados afirman llevar a cabo su práctica pedagógica. Destacando como las actividades más relevantes en más del $90.0 \%$ de los casos las asociadas con los ítems P1, P2 y P6 en donde los docentes aseguran presentar a sus estudiantes de forma clara los objetivos, conceptos e instrucciones de la clase ante cualquier actividad a realizar, empleando el adecuado lenguaje académico, siendo estos aspectos importantes ante cualquier clase o presentación de una temática nueva a abordar. Tal como lo afirma Villalobos (2011), "los objetivos deben estar acorde con las actividades que se plantean dentro de la unidad" (p.3). para ello los docentes en cada actividad deben establecer una relación tanto de contenidos, habilidades y actitudes que se espera desarrollar en los estudiantes. Contemplando una tarea fundamental del proceso de enseñanzaaprendizaje la formulación de objetivos adecuados en donde estos se den a conocer a los estudiantes. Es por esto que el logro de los objetivos es un eje principal de la planificación de clase (Reyes, 2017).

De la misma manera la presentación de las instrucciones de cada actividad es importante en nuestro proceso de enseñanza- aprendizaje pues facilitamos la comprensión de las actividades o temáticas que se están impartiendo. "Para lograr unas instrucciones claras es vital utilizar un lenguaje sin ambigüedades, tanto a nivel oral como escrito, de tal manera que sean comprensibles para los estudiantes y faciliten el proceso de enseñanza aprendizaje y la interrelación en el grupo" (Chávez, 2017, p.19). es por esto que el lenguaje académico influye en impartir instrucciones, pero este lenguaje no solo implica la acción verbal sino también sus gestos, escritura y movimientos; por esta razón para lograr presentar los objetivos, conceptos e instrucciones debemos ser coherentes en el lenguaje verbal y gestual que se utilice en el aula (Jiménez, Suárez \& Galindo, 2010).
También se observa que los docentes buscan estrategias para el proceso de enseñanza aprendizaje, en donde se destaca la motivación y la buena comunicación entre docente y estudiante, es por esto que el ítem P7 el $90.4 \%$ utiliza como estrategia incitar a sus estudiantes a la discusión de sus opiniones, pero utilizando adecuadamente el uso del lenguaje matemático, porque solo desde dicho conocimiento los estudiantes comprenderán los conceptos y contenidos esenciales en las matemáticas (Jaimes Ojeda, 2017). La importancia de utilizar el lenguaje formal en el aula, es que el estudiante propone ideas para mejorar o perfeccionar este idioma. Tal como lo expresa Brousseau (1986), el lenguaje y la comunicación trabajan de manera conjunta en el proceso de relación entre docente - estudiante, en donde por medio de estos se establecen interacción entre conceptos y procedimientos facilitando al desarrollo de la actividad matemática.

Así mismo en los ítems $\mathrm{P} 10$ y $\mathrm{P} 11$ se destaca la importancia de la evaluación escrita como recurso para identificar las oportunidades de mejora o fortalezas en este proceso, esto lo corrobora más del $90 \%$ de los docentes encuestados. Tal como lo afirma López (2003) la evaluación es una herramienta de transformación en la práctica del docente, puesto que devela las fortalezas, oportunidades de mejora y limitaciones del estudiante, con base en esta información los docentes pueden tomar acciones en su planeación y preparación de clases con el fin de garantizar el aprendizaje de los estudiantes.

Se identificó como una debilidad en los ítems de la práctica pedagógica que aproximadamente el $86.0 \%$ de los docentes encuestados aseguran algunas veces emplear aspectos relevantes en la práctica pedagógica, como es el caso del ítem P8 en donde el $34.2 \%$ de los docentes aseguran que algunas veces no proporcionan toda la información disponible 
con el fin de animar a los estudiantes a pensar por sí mismo, considerándose este como un aspecto importante de la comunicación que tiene el docente con el estudiante, por ende los docentes deben toman muy en cuenta en fortalecer este ítem e implementarlo en el aula de clase para no caer en una educación tradicional, repetitiva y aburrida para los educandos. De acuerdo con mi postura, Facione (1990) afirma, que el pensamiento crítico es la base de las matemáticas, es el punto de partida con el cual cada uno de los estudiantes aborda y empieza a razonar y a trabajar cada uno de sus pensamientos y habilidades.

\subsection{Influencia de los Años de experiencia del docente.}

Uno de los objetivos de esta investigación es determinar que influencia tienen los años de experiencia del docente con relación a las fortalezas y oportunidades de mejora de la práctica pedagógica, de acuerdo a esto se identificó que el $24.7 \%$ de los docentes tienen menos de 5 años de experiencia, el $20.5 \%$ entre 5 y 10 años, entre 11 y 20 años el $21.9 \%$ y por último el $31.9 \%$ de docentes encuestados tiene más de 20 años de experiencia, en vista de que la cantidad de docentes en cada intervalo es distinta se van a realizar tablas cruzadas pero totalizadas por filas con el fin de poder comparar los porcentajes de manera idónea. Para ello se realizaron estadísticas descriptivas y Chi-cuadrado para comparar si hay relaciones significativas entre ellas. Además, se muestra un comparativo entre los años de experiencia y otros ítems pertenecientes a las características sociodemográficas.

\begin{tabular}{|c|c|c|c|c|c|}
\hline & & \multicolumn{3}{|c|}{ Máximo nivel de formación académica } & \multirow[b]{2}{*}{ Total } \\
\hline \multicolumn{2}{|c|}{ Especialista } & Magister & Ninguno & & \\
\hline \multirow{4}{*}{$\begin{array}{l}\text { Años de } \\
\text { experiencia }\end{array}$} & $\begin{array}{l}\text { Menos de } 5 \\
\text { años }\end{array}$ & $5.5 \%$ & & $19.2 \%$ & $24.7 \%$ \\
\hline & $\begin{array}{l}\text { Entre } 5 \text { y } 10 \\
\text { años }\end{array}$ & $4.1 \%$ & $4.1 \%$ & $12.3 \%$ & $20.5 \%$ \\
\hline & $\begin{array}{c}\text { Entre } 11 \text { y } 20 \\
\text { años }\end{array}$ & $12.3 \%$ & $4.1 \%$ & $5.5 \%$ & $21.9 \%$ \\
\hline & $\begin{array}{l}\text { Más de } 20 \\
\text { años }\end{array}$ & $21.9 \%$ & $2.7 \%$ & $8.2 \%$ & $32.9 \%$ \\
\hline \multicolumn{2}{|c|}{ Total } & $43.8 \%$ & $11.0 \%$ & $45.2 \%$ & $100.0 \%$ \\
\hline
\end{tabular}

Aproximadamente el $25.0 \%$ de docentes encuestados tiene menos de 5 años de experiencia docente, el $19.2 \%$ de estos docentes no ha hecho ningún estudio pos gradual, esto indica que los docentes en sus 5 años de trabajo se dedican a satisfacer sus necesidades físicas o de confort, mientras que los que tienen entre 11 o más años de experiencia el $41.0 \%$ si han hecho estudios posgraduales y preferiblemente el fuerte son las especializaciones y no tanto las maestrías. La oferta académica de programas de posgrados en Cúcuta y su área metropolitana siempre se han focalizado fuertemente en el nivel de especialización y las maestrías son muy escasas y en las pocas que hay los costos son extremadamente elevados (Anzola, 2011). Por lo tanto, encontramos que los docentes acceden a lo que los recursos le permiten. 
Si bien en cierto, Colombia fue uno de los países que no consideraban necesario el posgrado, por ende, la formación de posgrados empezó a mediaos de la segunda mitad de siglo XX, en donde se comienzan a crear maestrías y tiempo después gestionan los primeros programas de doctorado, en la década de los setenta Colombia reconoce necesario la preparación de posgrados iniciando con un importante número de programas en el nivel de especialización y unos pocos en maestría y doctorado (Estratégica, 2006).En esta época eran pocos los docentes que realizaban actividades investigativas en las áreas fundamentales, pero al pasar los años se iban incorporando docentes con estudios pos graduales y experiencia investigativa que generaba controversia en aquellos docentes con formación de pregrado.

De acuerdo a lo anterior, un docente de calidad debe ser un excelente investigador para ello realiza este proceso en sus constantes actualizaciones accediendo a una educación posgradual como lo son maestrías y doctorados, se conoce que Colombia incremento los posgrados a nivel de especializaciones, así lo indican estadísticas registradas por el MEN, estando un poco alejadas estudios posgraduales a nivel de maestría y doctorado en donde en países como México y Brasil tomaron impulso y consolidaron que las especializaciones quedan en tercer plano siendo insignificantes en la formación del docente (Anzola, 2011).

Ahora bien, al analizar el aspecto económico un docente para acceder al posgrado en nivel intermedio como lo es la especialización debe contar con un total de 15 millones aproximadamente considerándose que este es uno de los modelos de más alto costo, para que el docente continúe con la maestría debe realizar una inversión de aproximadamente $\$ 40$ millones y por último el doctorado esta alrededor de los $\$ 80$ millones, para un total de $\$ 130$ millones. Considerando que estos costos son extremadamente altos y el docente no puede acceder a ellos, por ende, algunos docentes deciden formarse fuera del país. De acuerdo a los datos obtenidos podemos interpretar que los programas de especialización son más cortos, económicos y de menor exigencia académica que un programa de maestría y doctorado lo cual podría convertirse en un factor de motivación para que los docentes se inclinen más a hacer esos niveles que los programas de nivel avanzado. Pero después de cierto punto los docentes consideran que la especialización no resulta suficiente y quiere ascender a otro nivel mucho mayor y es ahí donde consideran los programas avanzados bien sea maestría y doctorados, es allí donde se identifica que el máximo nivel de formación está asociado con los años de experiencia.

Finalmente, un docente de calidad es aquel que está en constante actualización y auto superación, tomando como objetivo promover en los estudiantes aprendizajes significativos y conllevando a estos a una formación integral, así mismo el docente se convierte en un catalizador de cambio en donde por medio de sus estudios posgraduales logra indagar a profundidad las relaciones existentes entre el proceso de enseñanza-aprendizaje y pedagogía-didáctica, identificando los problemas de la educación y el contexto que rodea al estudiante redefiniendo así el saber pedagógico (Herrera, 2019). 
Tabla 4. Tabla de contingencia Años de experiencia vs Impulsa a sus estudiantes a participar haciendo uso del lenguaje preciso para profundizar en la comprensión de conceptos

\begin{tabular}{|c|c|c|c|c|c|}
\hline \multirow{2}{*}{\multicolumn{2}{|c|}{ Nunca }} & \multicolumn{4}{|c|}{$\begin{array}{l}\text { Impulsa a sus estudiantes a participar haciendo uso del } \\
\text { lenguaje preciso para profundizar en la comprensión de } \\
\text { conceptos }\end{array}$} \\
\hline & & Alqunas veces & Siempre & Total & \\
\hline \multirow{4}{*}{$\begin{array}{c}\text { Años de } \\
\text { experiencia }\end{array}$} & $\begin{array}{c}\text { Menos de } 5 \\
\text { años }\end{array}$ & $6.3 \%$ & $18.8 \%$ & $75.1 \%$ & $100.0 \%$ \\
\hline & $\begin{array}{c}\text { Entre } 5 \text { y } 10 \\
\text { años }\end{array}$ & & $16.7 \%$ & $83.4 \%$ & $100.0 \%$ \\
\hline & $\begin{array}{l}\text { Entre } 11 \text { y } 20 \\
\text { años }\end{array}$ & & $13.3 \%$ & $86.7 \%$ & $100.0 \%$ \\
\hline & $\begin{array}{l}\text { Más de } 20 \\
\text { años }\end{array}$ & & $8.3 \%$ & $91.7 \%$ & $100.0 \%$ \\
\hline \multicolumn{2}{|c|}{ Total } & $1.4 \%$ & $13.7 \%$ & $84.9 \%$ & $100.0 \%$ \\
\hline
\end{tabular}

Fuente: Elaboración propia

Al indagar entre los docentes encuestados se encontró a nivel general, que el $84.9 \%$ de ellos afirma que siempre impulsan a sus estudiantes a participar haciendo uso del lenguaje preciso para profundizar en la comprensión de conceptos, como docente en formación considero que uno de los elementos que conlleva al mejoramiento del aprendizaje es la participación y motivación que se emplee en la clase, ahora bien al abordar una clase el docente debe hacer uso del lenguaje oral y escrito de manera adecuada para que conlleve a sus estudiantes a la comprensión de conceptos y una mayor participación en el aula, siendo este un aspecto importante en la comprensión de los conceptos matemáticos, tal como lo afirma Ponte et al. (2007, como se citó en Jiménez, Suarez y Galindo, 2010)

el lenguaje oral sirve de soporte al pensamiento e, incluso, es a través de él que se desarrollan los aspectos esenciales de la enseñanza y el aprendizaje de la matemática; sin embargo, el lenguaje escrito, incluyendo todo tipo de registro escrito, simbólico o representación iconográfica, es una forma complementaria de comunicación y un medio importante que permite a los alumnos reflexiohar sobre su comprensión matemática, y hacer explícitas las conexiones entre diversos conceptos (pg.11).

Al indagar con relación a los años de experiencia, se tiene que el $91.7 \%$ de los docentes con más de 20 años de experiencia manifiesta que siempre impulsan a sus estudiantes a participar haciendo uso de un lenguaje adecuado para la comprensión de conceptos. Se destaca como fortaleza que así el docente de matemáticas sea recién graduado o con más de 20 años de experiencia siempre promociona en sus estudiantes el uso de lenguaje formal propio de la matemática identificándose que este va aumentando debido a sus años de experiencia. 
Tabla 5. Tabla de contingencia Años de experiencia vs Motiva a sus estudiantes a la formulación de preguntas de forma individual o grupal, como recurso de apoyo en el aula

\begin{tabular}{|c|c|c|c|c|c|}
\hline \multirow{2}{*}{\multicolumn{2}{|c|}{ Nunca }} & \multicolumn{4}{|c|}{$\begin{array}{l}\text { Motiva a sus estudiantes a la formulación de preguntas de forma } \\
\text { individual o grupal, como recurso de apoyo en el aula }\end{array}$} \\
\hline & & Algunas veces & Siempre & Total & \\
\hline \multirow{4}{*}{$\begin{array}{l}\text { Años de } \\
\text { experiencia }\end{array}$} & $\begin{array}{l}\text { Menos de } 5 \\
\text { años }\end{array}$ & & $31.3 \%$ & $68.8 \%$ & $100.0 \%$ \\
\hline & $\begin{array}{l}\text { Entre } 5 \text { y } 10 \\
\text { años }\end{array}$ & $11.1 \%$ & $11.1 \%$ & $77.7 \%$ & $100.0 \%$ \\
\hline & $\begin{array}{l}\text { Entre } 11 \text { y } 20 \\
\text { años }\end{array}$ & & $6.7 \%$ & $93.4 \%$ & $100.0 \%$ \\
\hline & $\begin{array}{l}\text { Más de } 20 \\
\text { años }\end{array}$ & & & $100.0 \%$ & $100.0 \%$ \\
\hline \multicolumn{2}{|c|}{ Total } & $2.7 \%$ & $11.0 \%$ & $86.3 \%$ & $100.0 \%$ \\
\hline
\end{tabular}

Al indagar entre los informantes se encontró a nivel general, que el $86,3 \%$ de ellos siempre motivan a sus estudiantes a la formulación de preguntas de forma individual o grupal, como

recurso de apoyo en el aula, considerando los beneficios que se obtienen tales como: descubrir las dificultades del proceso de aprendizaje, ayudar a que el estudiante piense críticamente y orientar, motivar y validar el conocimiento que tiene para dar respuesta a los interrogantes que se presenten en el aula de clase. respecto a lo anterior Jiménez, Suarez y Galindo (2010) considera que,

el aula se convierte en un ambiente de aprendizaje que favorece la discusión, la pregunta, la concertación y la negociación de significados, y que al maestro le corresponde propiciar el ambiente interactivo del aula, de tal manera que tanto docente como estudiantes sean interlocutores activos en un espacio vivo (p.18).

Es por ello que mediante la formulación de preguntas el estudiante no solo aportara a su aprendizaje individual, sino que creara una interacción grupal, pues a partir de cada idea se puede validar y construir de manera colectiva con el aprendizaje de sus compañeros. Al indagar la correlación con respecto a los años de experiencia resulta significativo mencionar que el $100 \%$ de los docentes con más de 20 años de experiencia siempre motivan a sus estudiantes a trabajar la formulación de preguntas de manera colectiva empleando este como un recurso a su clase, contrario a esto el $31.3 \%$ de los docentes con menos de 5 años de experiencia algunas veces emplean este ítem, identificando que el docente recién graduado no dimensiona el valor de la pregunta y en el momento que va adquiriendo experiencia va reflexionando que la pregunta sirve para hacer control de atención y para hacer control del entendimiento de conceptos, por ende el porcentaje va ascendiendo en función a los años de experiencia. Así mismo los docentes con más de 20 años reconocen y están absolutamente convencidos que la pregunta es un recurso interesante para motivar y escuchar lo que el estudiante piensa y la manera como ha interpretado y adquirido el conocimiento. 
Tabla 6. Tabla de contingencia Años de experiencia vs En el proceso de seguimiento del aprendizaje de los estudiantes, recurre a la retroalimentación para hacer correcciones cuando es necesario

\begin{tabular}{|c|c|c|c|c|}
\hline \multirow{2}{*}{\multicolumn{2}{|c|}{ Algunas veces }} & \multicolumn{3}{|c|}{$\begin{array}{l}\text { En el proceso de seguimiento del aprendizaje de los } \\
\text { estudiantes, recurre a la retroalimentación para hacer } \\
\text { correcciones cuando es necesario }\end{array}$} \\
\hline & & Siempre & Total & \\
\hline \multirow{4}{*}{$\begin{array}{c}\text { Años de } \\
\text { experiencia }\end{array}$} & Menos de 5 años & $25,0 \%$ & $75,1 \%$ & $100,0 \%$ \\
\hline & $\begin{array}{l}\text { Entre } 5 \text { y } 10 \\
\text { años }\end{array}$ & $22,2 \%$ & $77,8 \%$ & $100,0 \%$ \\
\hline & $\begin{array}{c}\text { Entre } 11 \text { y } 20 \\
\text { años }\end{array}$ & & $100,0 \%$ & $100,0 \%$ \\
\hline & Más de 20 años & & $100,0 \%$ & $100,0 \%$ \\
\hline \multicolumn{2}{|c|}{ Total } & $11,0 \%$ & $89,1 \%$ & $100,0 \%$ \\
\hline
\end{tabular}

Con respecto a lo observado se tiene que el $89,1 \%$ de los docentes encuestados manifiestan que siempre en el proceso de seguimiento del aprendizaje recurren a la retroalimentación, conociendo que el docente siempre busca estrategias para que el aprendizaje sea significativo y todo esto se da partir del monitoreo de sus clases y la interacción que tiene en estas, por ello la retroalimentación es clave en este proceso pues ayuda a que el estudiante comprenda en que aspecto está fallando y que conocimiento tiene herrado, mejorando así su aprendizaje y diferenciando las ideas que tenía a las que tiene después de clarificar sus dudas, también esta herramienta como lo es la retroalimentación ayuda a que el docente reflexione y explore la efectividad de sus prácticas y reoriente todo su proceso de enseñanza formando con esto estudiantes competentes capaces de analizar las situaciones de su diario vivir ( Martínez \& Vargas, 2014).

Con respecto a los años de experiencia del docente se encontró que el $100.0 \%$ de los docentes pertenecientes entre 11 y más de 20 años siempre recurren a la retroalimentación como recurso en el proceso de seguimiento de sus estudiantes, también podemos analizar que el $47 \%$ de docentes entre 0 y 10 años de experiencia algunas veces incorporan la retroalimentación como recurso del proceso de seguimiento. Pero se destaca que es poca la diferencia entre cada uno de ellos evidenciándose que por medio del seguimiento el estudiante obtendrá un aprendizaje significativo.

\subsection{Fortalezas y oportunidades de mejora de la práctica pedagógica}

Para identificar las fortalezas y oportunidades de mejora de la práctica pedagógica y establecer correlaciones significativas entre cada uno de los ítems se realizaron aproximadamente 373 pruebas, de las cuales en 225 se logró evidenciar que si existía un vínculo entre estos aspectos. Al analizar los distintos ítems al interior de cada una de las subcategorías dentro de la dimensión de la practica pedagógica se pudo verificar que los docentes afirman que dentro del ejercicio profesional incorporan y tiene en cuenta varios aspectos que consideran fundamentales. Algunos de estos son los que se muestran a continuación.

a) Los docentes encuestados tienen clara las relaciones entre los conceptos matemáticos que enseña identificando cuales son 
prerrequisitos de otros siendo fundamental tener dominio del tema que se está enseñando, este dominio permitirá que el estudiante comprenda el tema más allá del soporte didáctico que se utilice. Además, al tener claro las relaciones existentes entre los conceptos se logra que el estudiante construya su propio conocimiento relacionando los conceptos que aprende y abordándolos por medio de estructuras conceptuales que posee, generando en ellos un aprendizaje significativo.

Tal como lo afirma Gómez, Acuña \& Romero (2005),

mientras captamos información estamos constantemente organizándola en unidades con algún tipo de ordenación, que llamamos estructura. Ausubel plantea que el aprendizaje del alumno depende de la estructura cognitiva previa que se relaciona con la nueva información y debe entenderse por "estructura cognitiva" (p.17).

Por lo tanto, el docente debe propiciar la construcción de esquemas en donde el estudiante deberá modificarlos y enriquecerlos facilitando así un aprendizaje con significados.

b) Ocasionalmente el docente, no proporciona toda la información disponible con el fin de animar a los estudiantes a pensar por sí mismo. Siendo este un aspecto súper importante a resaltar debido a que en el trascurso del tiempo la educación ha sido concebida como una simple transmisión de conocimientos, siendo obsoletos antes de ser asimilados por los estudiantes, por eso es importante que el estudiante sea el responsable de su propio proceso de aprendizaje, en donde el mismo construya su propio conocimiento, estableciendo así que la función del docente es orientar esta actividad de construcción en donde el estudiante se acerque progresivamente al significado y representación de cada contenido. Tal como lo afirma Tesouro (2005),

la función del educador no tiene que ser la de transmitir conocimientos, sino la de proporcionar al alumno sistemas de codificación de la información amplios y funcionales, para que posibiliten un aprendizaje inteligente, productivo $y$ creativo que facilite la adaptación a la gran cantidad de peticiones del medio y a su variabilidad (p.6).

Por lo tanto, el docente debe centrarse en enseñar a pensar y fomentar la capacidad de raciocinio de cada uno de los estudiantes, en donde cultive en ellos un pensamiento crítico logrando así una educación de calidad.

c) El docente establece las rutinas de clase que permiten el buen funcionamiento de esta. Siendo esta un ítem perteneciente a los ambientes de aprendizaje, se es bien conocido que los docentes deben buscar herramientas pedagógicas y didácticas con las cuales respondan al reto de educar en el mundo de hoy en donde por medio de estas mejore y garantice el aprendizaje de los estudiantes es por ello que las rutinas de clase es una gran alternativa tal como lo afirma Grenier (2018),

la incorporación de las rutinas escolares al aula, ha permitido que los estudiantes logren un uso eficiente del tiempo y hacer transiciones tranquilas entre las diferentes actividades que se desarrollan en clase, en la medida que ellos se familiarizan con momentos en los cuales cumplen claramente por medio de acuerdos $y$ procedimientos constantes, sencillos $y$ claros, que les permiten conocer qué se espera de ellos, independizándose de la figura del profesor como una única fuente de aprendizaje. (p.2) 
Es por esto que establecer rutinas de clase genera un buen clima en el aula generando un ambiente de respeto, una buena organización del espacio físico y se valora todo lo que se aprende día a día.

d) Los docentes presentan los conceptos con precisión, claridad e imaginación utilizando el lenguaje académico adecuado, considerándose uno de los aspectos fundamentales para la presentación de una nueva temática a abordar que involucra la relación entre docente estudiante Tal como lo afirma Ponte et al. (1997, como se citó en Jiménez, Suarez y Galindo, 2010)

la comunicación se refiere a la interacción entre los diversos sujetos que hay en una clase, empleando un lenguaje propio, que es una mezcla del lenguaje cotidiano y del matemático. Cuando hay interacción comunicativa, la negociación de significados aparece de manera natural, la cual se refiere al modo en que los alumnos y el profesor exponen unos a otros su forma de entender los conceptos y los procesos matemáticos, los perfeccionan y los ajustan al conocimiento matemático (p.8).

Así pues, en el proceso de enseñanzaaprendizaje la comunicación es uno de los factores fundamentales para establecer una buena relación con el estudiante en donde se trabaje de manera armónica y este proceso sea más ameno tanto para ellos como para el docente, esto conlleva que el estudiante obtenga aprendizajes significativos, puesto que si existe una buena comunicación el docente se expresara libremente empleando un lenguaje adecuado y lleva sus estudiantes a una educación de calidad.

\section{CONCLUSIONES}

En el trayecto de esta investigación se ha analizado las posibles oportunidades de mejora y fortalezas de la practica pedagógica del docente matemático en función a los años de experiencia, por consiguiente, se determinó si la practica pedagógica se correlaciona con los años de experiencia y a su vez si existía correlación entre todas las dimensiones que debe abordar el docente en su práctica pedagógica, siendo estas las mencionadas en el Marco de Desarrollo Profesoral (2013).

Con respecto a los resultados encontrados, se destaca que los años de experiencia tiene una correlación significativa con el máximo nivel de formación, evidenciándose que son muchos los docentes que no ingresan a realizar una formación avanzada específicamente los programas de maestría y doctorado identificando diversos factores como lo son la falta de tiempo y los altos costos de cada programa, por ello los docentes en sus primeros años de experiencia se dedican a satisfacer sus necesidades básicas y tiempo después es que consideran realizar un formación posgradual inclinándose por el nivel intermedio debido a que es más sencillo en cuanto a tiempo, es más asequible en cuanto a costo y el nivel de exigencia académica es menor. Pasados los 15 años de experiencia el docente considera ascender en su formación posgradual debido a que la especialización no le resulta suficiente.

Así mismo, se evidencio que las prácticas pedagógicas están fuertemente relacionadas con los años de experiencia debido a esto, el docente no tiene en cuenta muchos aspectos en el aula tanto en los ambientes de aprendizaje como en la planeación y preparación de esta.

De acuerdo lo anterior los docentes recién graduados son aquellos que no saben identificar o no dimensiona la importancia de cada uno de estos dentro del proceso de enseñanza, a 
medida que van adquiriendo experiencia van identificando la importancia de potenciar cada una de las dimensiones que involucra la práctica pedagógica.

Ahora bien, para identificar que fortalezas y oportunidades de mejora estaban presentes en la práctica pedagógica del docente, se realizaron diversas correlaciones entre las dimensiones que involucra la propia practica siendo estos ambientes de aprendizaje y planeación y preparación de clase. Se pone de manifiesto que fueron muchos los ítems que estaban significativamente correlacionados en cuanto a la prueba de independencia chi-cuadrado, así mismo se identificó que no existe ninguna debilidad en cuanto a la práctica pedagógica del docente matemático, caso contrario a sus fortalezas destacándose muchos ítems que son esenciales dentro del ejercicio profesional.

Por ende, se destaca en todo este proceso de análisis que el docente no solo se desempeña en brindar contenidos, si no que su función como docente es dedicar ese conocimiento acompañado de la pedagogía que se requiera para resignificar el contexto en el que se encuentra el estudiante. Para que el docente cumpla con todas las dimensiones que competen una práctica pedagógica de calidad debe saber utilizar la didáctica en función a los saberes (ser-hacer y conocer) obteniendo competencias de trabajo en equipo, liderazgo, comunicación, entre otras.

Finalmente se concluye en esta investigación que las dimensiones de la práctica pedagógica no pueden funcionar de manera independiente y aislada unos de otros. siempre debe abordarse la practica pedagógica con todas las dimensiones, es decir para que un docente fomente una educación de calidad, debe abordar en su práctica pedagógica los ambientes de aprendizaje y la planeación y preparación de clase, para que lleve a los educandos a alcanzar su formación integral y brindar así un aprendizaje significativo.

Se sugiere para futuras investigaciones posteriores el hacer un ejercicio complementario de observación o de verificación de lo que los docentes están afirmando. Debido a que hasta el momento estamos tomando la opinión de los docentes con respecto a la aplicación de la encuesta, por ende, el realizar un ejercicio de acompañamiento o de observación de la práctica del docente y verificación del material que utiliza en clase se conllevaría a analizar a fondo si existe alguna debilidad $u$ otras fortalezas por resaltar en su proceso de enseñanza.

\section{REFERENCIAS BIBLIOGRAFICAS}

Anzola Montero, G. (2011). Realidad de los posgrados en Colombia y su situación frente a la reforma de la Ley 30 . Revista UDCA. Actualidad \& Divulgación Científica, 14(2), 3-5. Recuperado de: $\quad$ http://www.scielo.org.co/scielo. php? script $=$ sci_arttext $\&$ pid $=$ S0123$42262011000200001 \&$ Ing=en\&tlng=es.

Basurto, J. G. R., Hernández, E. R., \& Rodríguez, Y. E. R. (2013). El trabajo docente: Una mirada para la reflexión. Perspectivas docentes, (51), 3538. Recuperado de: https://dialnet.unirioja. es/servlet/articulo?codigo $=6349290$

Brousseau, G. (1983). Obstacles épistémologiques et les problémes en mathématiques. Recherches en Didactique des Mathématiques, 4(2), 165-198.

Chávez, N. J. (2017). Instrucciones en el aula. Revista universitaria de informática. RUNIN, 2(4), 18-21.

Danielson, C. (2013). A Framework for Teaching. Alexandria, VA: Association for Supervision and Curriculum Development. 
Duque, P. A., Vallejo, S. L., \& Rodriguez, J. C. (2016). Prácticas pedagógicas y su relación con el desempeño académico (Master's thesis).

Estratégica, O. (2006). La oferta y la demanda de formación avanzada en Colombia. Estudio técnico presentado al DNP ya Colciencias.

Facione, Peter A. (1990). Executive summary of critical thinking: A statement of expert consensus for purposes of educational assessment and instruction, Berkeley: The California Academic Press.

Freire, P. (2011). Cartas a quien pretende enseñar. siglo XXI editores. Buenos Aires.

Gamboa, A. (2016). Calidad de la educación superior: pretensiones y realidades institucionales: Bogotá, Colombia: Ecoe.

García-Quintero, C., \& Villamizar Suárez, G. (2017). Análisis fenomenológico de la conciencia del docente a partir de sus prácticas evaluativas. Revista Perspectivas, 2(2), 49-59. https://doi. org/10.22463/25909215.1313.

Herrera Pérez, J. C. (2019). Formación docente a nivel de postgrado en Latinoamérica.

Jaimes- Ojeda, L. (2017). Propuesta metodológica para la enseñanza de la química en la Educación Media apoyada en el aprendizaje basado en problemas (APB). Revista Perspectivas, 2(2), 6-16. https:// doi.org/10.22463/25909215.1310.

Jiménez, A., Suárez, N. Y., \& Galindo, S. M. (2010). La comunicación: eje en la clase de matemáticas. Praxis \& Saber, 1(2), 173202.

López, A. A. (2014). La evaluación como herramienta para el aprendizaje: conceptos, estrategias y recomendaciones. Magisterio. Mathématiques, 72, 33-115. GrenobleFrance: La pensée sauvage.
Lago, D., Gamboa, A., Montes, A. (2014). Calidad de la educación superior: un análisis de sus principales determinantes. Saber, ciencia y libertad, 9(1), 157-170. Recuperado de: https://dialnet.unirioja.es/servlet/ articulo?codigo $=5104971$

María Isabella Grenier. (2018). Las rutinas escolares como herramienta pedagógica y didáctica del docente para mejorar el aprendizaje de los estudiantes del siglo XXI Programa Todos a Aprender (PTA). Bogotá, Colombia.

Martínez, F. G. L., \& Vargas, L. A. T. (2014). Retroalimentación formativa para estudiantes de educación a distancia. RIED. Revista Iberoamericana de Educación a Distancia, 17(2), 197-221.

Martínez, Y. (2017). La reflexión de la práctica pedagógica: un camino a transitar en la construcción de saber pedagógico. Análisis del Proyecto de Aula "Cosmos: Una aventura Interplanetaria"-IPARM UN.

Ministerio de educación nacional (MEN). (2005). Revolución educativa, altablero. bogotá, colombia. Recuperado de https://www.mineducacion.gov.co/1621/ propertyvalues-31232_tablero_pdf.pdf

Ministerio de educación nacional (MEN). (2013). Marco profesoral. Bogotá, Colombia. Recuperado de http://aprende. colombiaaprende.edu.co/sites/default/files/ naspublic/anexo 3 - marco para la ensenanza.pdf

Morillo Carlosama, O. A. (2014). Concepciones y prácticas docentes sobre la diversidad como base para la innovación pedagógica. Un estudio de caso en el colegio Vasco Núñez de Balboa.

Pacheco, M. C. A. C., \& Ramos, M. E. F. (2015). La calidad de la Educación, una problemática colombiana - The quality of education, a problema colombian. Revista Edu-Física, 7(16). 
Penagos, M., Mariño, L. F., \& Hernández, R. V. (2017). Pensamiento matemático elemental y avanzado como actividad humana en permanente evolución. Revista Perspectivas, 2(1), 105-116. https://doi. org/10.22463/25909215.1289.

Pérez Montes, D. (2018). La reflexión de nuestra propia práctica docente para mejorar el proceso lector de las estudiantes (Masteris thesis, Universidad del Norte).

Piñero, J. C. (2020). Modelando los diferentes roles del docente en la educación matemática moderna. Espacios, 41(30), 301-317.

Ponte, J.P. Da et al. (2007). "A comunicação nas práticas de jovens professores de Matemática". Revista Portuguesa de Educação, 20(2): 39-74. Universidade de Lisboa, Faculdade de Ciências. Recuperado el 09/08/2010 de: http://www. educ.fc.ul/docentes/jponte.

Reyes-Salvador, J. (2017). La planeación de clase; una tarea fundamental en el trabajo docente. Maestro y sociedad, 14(1), 87-96.

Rodríguez, M. E. (2010). El perfil del docente de matemática: visión desde la triada matemática-cotidianidad y pedagogía integral/The mathematics teacher profile: a perspective from the "mathematics, daily life experiences and integral pedagogy" triad. Actualidades investigativas en educación, 10(3).

Tesouro, M. (2005). La metacognición en la escuela: la importancia de enseñar a pensar. Educar, 35, 135-144.

Villalobos, X. (2011). Reflexión en torno a la gestión de aula ya la mejora en los procesos de enseñanza y aprendizajes. Revista iberoamericana de educación, 55(3), 1.

Zilberstein, J., Silvestre, M. \& Olmedo, S. (2016). Diagnóstico y transformación de la institución docente. México: Ediciones CEIDE. 